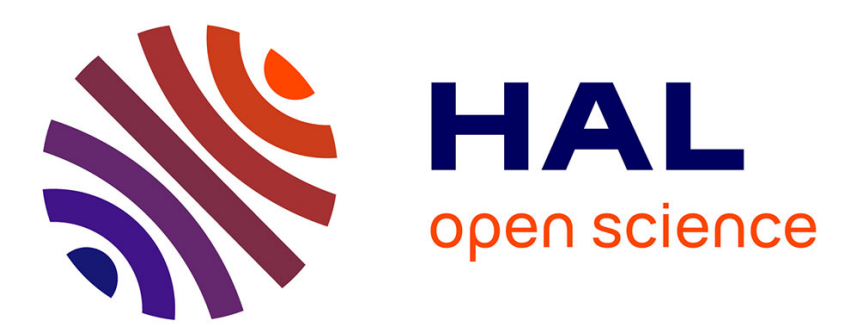

\title{
Enhanced multiple ultrasonic shear reflection method for the determination of high frequency viscoelastic properties
}

\author{
Viviane Cereser Camara, Didier Laux, Olivier Arnould
}

\section{To cite this version:}

Viviane Cereser Camara, Didier Laux, Olivier Arnould. Enhanced multiple ultrasonic shear reflection method for the determination of high frequency viscoelastic properties. Ultrasonics, 2010, 50, pp.710715. 10.1016/j.ultras.2010.02.007 . hal-00689413

HAL Id: hal-00689413 https://hal.science/hal-00689413

Submitted on 19 Apr 2012

HAL is a multi-disciplinary open access archive for the deposit and dissemination of scientific research documents, whether they are published or not. The documents may come from teaching and research institutions in France or abroad, or from public or private research centers.
L'archive ouverte pluridisciplinaire HAL, est destinée au dépôt et à la diffusion de documents scientifiques de niveau recherche, publiés ou non, émanant des établissements d'enseignement et de recherche français ou étrangers, des laboratoires publics ou privés. 


\title{
Enhanced multiple ultrasonic shear reflection method for the determination of high frequency viscoelastic properties
}

\author{
V. Cereser Camara ${ }^{\text {a }}$, D. Laux ${ }^{\mathrm{a}, *}$, O. Arnould ${ }^{\mathrm{b}}$ \\ a Institut d'Electronique du Sud, IES, UMR CNRS 5214, Université Montpellier II, CC 082, Place Eugène Bataillon, 34095 Montpellier, Cedex 5, France

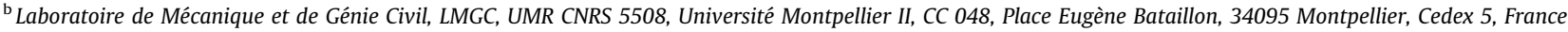

\section{A R T I C L E I N F O}

\section{Article history:}

Received 22 June 2009

Received in revised form 11 February 2010

Accepted 11 February 2010

Available online 17 February 2010

\section{Keywords:}

Viscoelasticity

Multiple ultrasonic shear wave reflection method

Acoustic impedance

Glycerin

Newtonian behaviour

\begin{abstract}
A B S T R A C T
In this work, we propose a study dedicated to the influence of the delay line nature in transverse ultrasonic sensors, dedicated to dynamic high frequency elastic moduli of viscoelastic materials estimation. In literature, these shear ultrasonic rheometers are using delay lines in glass or quartz and normal or oblique incidence of ultrasonic rays. The oblique incidence is used in order to improve the sensitivity of the measurements. We theoretically demonstrate in this work that the use of delay lines in polymers is recommended to improve the sensitivity. Due to modifications, performed on a $10 \mathrm{MHz}$ commercial ultrasonic sensor, we experimentally show on glycerin (which is a Newtonian material) that it is possible to multiply by a factor 10 the sensitivity; compared to delay lines in quartz using a normal incidence of rays. Hence, we overpass the accuracy of the oblique incidence approach with a simpler experimental setup.

(c) 2010 Elsevier B.V. All rights reserved.
\end{abstract}

\section{Introduction}

Measurement of material properties using ultrasound has intensively been studied for decades [1]. This technique has been demonstrated to be very efficient for nondestructive examination. The advantage of this method is the direct coupling between ultrasonic quantities and mechanical properties. So, ultrasonic measurements can be considered as continuation of rheology for high frequencies.

In the low frequency range $(\mathrm{Hz}-\mathrm{kHz})$, the viscoelastic properties are usually determined by DMA (Dynamic Mechanical Analysis) or rheology. In the ultrasonic frequency range $(\mathrm{kHz}-\mathrm{GHz})$, several experimental techniques (ultrasonic pulse transmission technique, thickness shear mode, Bulk acoustic wave, ultrasonic shear wave reflected method, etc.) based on longitudinal or shear waves already exist [2-10]. However, all these techniques present some limitations. Indeed, to study the properties of soft viscoelastic materials, it is difficult to use the ultrasonic pulse transmission technique because of the extremely high attenuation of shear waves propagation in these materials. Resonant methods transform a measured quantity into a frequency shift of an electrical signal. These devices can be used to characterize the mechanical behaviour of materials bonded on their surface.

\footnotetext{
* Corresponding author.

E-mail addresses: didier.laux@ies.univ-montp2.fr, laux@lain.univ-montp2.fr (D. Laux)
}

With such methods, it is necessary to use an algorithm which enables the conversion of the raw data of the impedance into equivalent circuit parameters. Therefore, the dynamic moduli cannot directly been determined with these methods. That is the main drawback of these methods compared to the technique proposed by O'Neil and Mason [11,12]. This latter has been proposed in 1949 in order to compare two types of ultrasonic reflectivity measurements using normal or oblique incident shear waves. The technique uses the complex reflection coefficient (modulus and phase) at an interface between a buffer rod and the material to determine the viscoelastic properties (dynamic moduli, $G^{\prime}$ and $G^{\prime \prime}$ ) of the material under study. The advantage of the oblique incidence method compared to the normal incidence one is its sensitivity: about 5 times better. Concerning the diffraction effects, the normal incidence wave method requires a shorter buffer rod (around $7 \mathrm{~mm}$ ) than for the oblique incidence method (around $80 \mathrm{~mm}$ ) [13]. So, a normal incidence technique seems preferable.

The aim of this work is to demonstrate that using the previously presented ultrasonic shear wave technique in normal incidence and at the cost of some modifications performed on a commercial sensor in order to adapt a delay line in polymer, one can reach a sensitivity at least equal to the one obtained with the same technique in oblique incidence.

The paper is organized as follows: the theoretical backgrounds of ultrasonic shear wave reflection method are presented in Section 2. In particular, we will focus our attention on the influence 
of delay line on the experimental quantities to be measured and which directly affect the sensitivity of the system. Section 3 describes the design of the transducer. Section 4 presents the experimental setup. At last, the results are presented and commented in Section 5. In particular the effect of delay line attenuation is discussed.

\section{Theory}

\subsection{Ultrasonic shear wave reflection theory [1,11,12]}

According to the theory of wave reflection, for the normal incidence of shear waves at a boundary between two elastic materials, the reflection coefficient can be defined as:

$r=\frac{A_{\text {reflected }}}{A_{\text {incident }}}=\frac{Z_{2}-Z_{1}}{Z_{2}+Z_{1}}$

where $r$ is the wave reflection coefficient in pressure or stress, defined as the amplitude ratio between the reflected and incident waves, which can be obtained directly from experimental measurements. $Z_{1}$ and $Z_{2}$ are the shear acoustical impedances of the materials. This acoustical impedance is defined as the product (density) $\times$ (shear velocity). In the case of pure elastic materials, acoustical impedance and $r$ are real parameters. If the viscoelastic behaviour is considered, the acoustical impedance is also a complex quantity (Eq. (2)). Considering now that the material 1 is elastic and that the material 2 is viscoelastic, reflection coefficient can be generalized in a complex format:

$Z^{*}=R+j X$

$r^{*}=r_{0} e^{i \Phi}=r_{0}(\cos \Phi+j \sin \Phi)$

where $\phi$ is the phase shift between the incident and the reflected wave, and $r_{0}$ is the magnitude or modulus of the reflection coefficient. This means that, when an ultrasonic wave propagating in material 1 is reflected on the interface ( $1 / 2)$, its amplitude decreases and the wave undergoes a phase shift.

The real $(R)$ and imaginary part $(X)$ of the shear impedance of the viscoelastic material, given in Eqs. (4) and (5); can be obtained using Eqs. (2) and (3)

$R=Z_{1} \frac{1-r_{0}^{2}}{1-2 r_{0} \cos \Phi+r_{0}^{2}}$

$X=Z_{1} \frac{2 r_{0} \sin \Phi}{1-2 r_{0} \cos \Phi+r_{0}^{2}}$

For ultrasonic shear waves, the complex acoustic impedance $Z^{*}$ is related to the storage $\left(G^{\prime}\right)$ and the loss $\left(G^{\prime \prime}\right)$ moduli through the following relationship:

$Z^{*}=\sqrt{\rho G^{*}}=\left(\rho\left(G^{\prime}+j G^{\prime \prime}\right)\right)^{1 / 2}$,

where $\rho$ is the density of the material studied. Both the storage and loss shear modulus can be expressed as a function of the real and imaginary part of the shear impedance as shown in Eqs (7) and (8).

$$
\begin{aligned}
G^{\prime} & =\frac{R^{2}-X^{2}}{\rho} \\
G^{\prime \prime} & =\frac{2 R X}{\rho}
\end{aligned}
$$

We can rewrite the storage and loss shear modulus by replacing the Eqs. (4) and (5) in the Eqs. (7) and (8), where $\rho$ is the density of the studied material, $\rho_{\mathrm{DL}}$ the density of the delay line and $V_{D L}$ the shear ultrasonic velocity in the delay line.

$$
\begin{aligned}
G^{\prime} & =\left(\rho_{D L} V_{D L}\right)^{2} \frac{\left(r_{0}^{2}-1\right)^{2}-4 r_{0}^{2} \sin ^{2} \Phi}{\rho\left(2 r_{0} \cos \Phi+1+r_{0}^{2}\right)^{2}} \\
G^{\prime \prime} & =-4\left(\rho_{D L} V_{D L}\right)^{2} \frac{\left(r_{0}^{2}-1\right) 4 r_{0} \sin \Phi}{\rho\left(2 r_{0} \cos \Phi+1+r_{0}^{2}\right)^{2}}
\end{aligned}
$$

Experimentally, an ultrasonic transducer made of a piezoelectric crystal and a delay line in silica or glass for instance, is used. It represents the material 1 in the previous paragraph. The material 2 is constituted by the polymer which is studied. For some polymers the phase shift undergone by the ultrasonic wave is measurable on the first echo reflected on the viscoelastic interface. Hence, the method can be used. We can cite studies of polydimethylsiloxane polymers and curing epoxy systems $[14,15]$.

But, in many cases, the phase shift is so small that no measurement is possible on the first echo. But, as the ultrasonic attenuation is small in the delay line, multiple reflections are possible leading to the existence of many echoes. Using this fact, Gasparoux et al. [16] have proposed a multiple ultrasonic reflection device (MUR). They worked with the multiple reflections instead of a simple one, at the interface between the delay line and the material. Then, the complex reflection coefficient, $r^{*}$ was calculated with the following relationships, where: $A_{n}$ is the amplitude of the echo number $n$, in the case of the interface (delay line/air), $B_{n}$, the amplitude for the interface (delay line/material), $\Delta t_{n}$ the time shift between these two echoes and $f$ the operating frequency (see Fig. 1).

$r_{o}=\left(\frac{B_{n}}{A_{n}}\right)^{\frac{1}{n}}$
$\Phi=-2 \pi f\left(\frac{\Delta t_{n}}{n}\right)$

If $n$ echoes are used, the time-lag or phase shift to be measured, is multiplied by a factor $n$.

All the theory pointed above has considered the impedance of the delay line as a real number. Considering now that the materials 1 and 2 are viscoelastic, and taking into account the attenuation, we can develop a complex impedance theory.

Let $Z^{*}$ and $k^{*}$ complex impedance and wave vector respectively in the delay line which is now considered as a damping material. The Eq. (2) can be rewritten as following:

$Z^{*}=\rho_{D L} \cdot \frac{\omega}{k^{*}}$

$k^{*}=\frac{\omega}{v_{s}}-j \cdot \alpha$

In these expressions, $\alpha$ is the ultrasonic wave attenuation and $\omega$ the radian frequency $(\omega=2 \Pi f)$. Then, $Z^{*}$ can be written as follows:

$Z^{*}=R+j X=\rho_{D L}(c+j \cdot b)$,

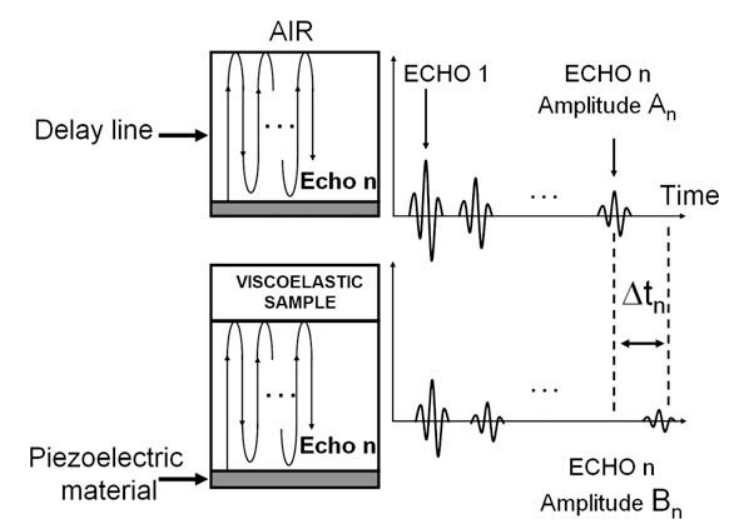

Fig. 1. Schematic representation of the multiple ultrasonic reflection method. 


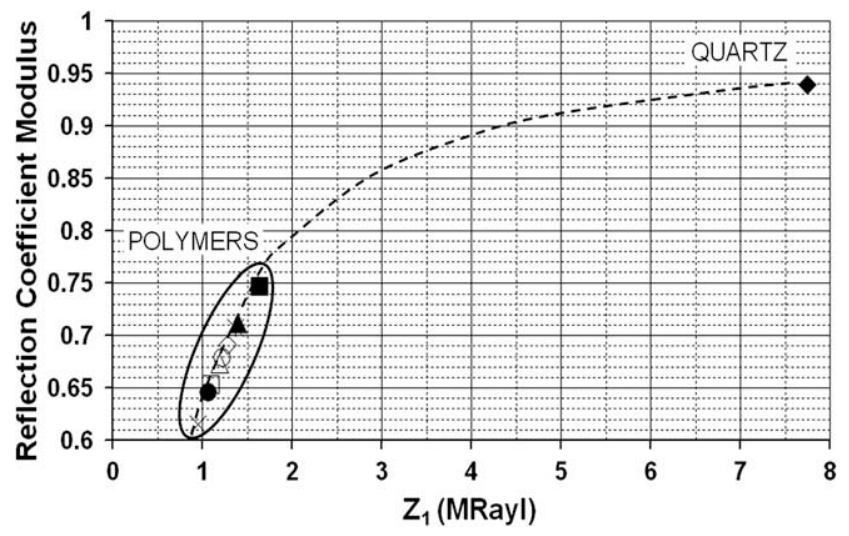

Fig. 4a. Reflection coefficient simulation at $10 \mathrm{MHz}$ as a function of the delay line materials on glycerin at room temperature $\left(20^{\circ} \mathrm{C}\right)$.

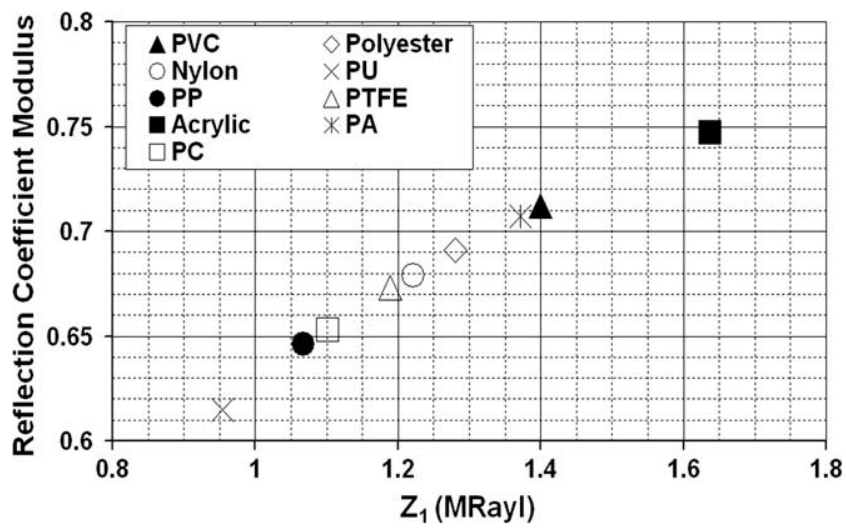

Fig. 4b. Zoom of reflection coefficient simulation at $10 \mathrm{MHz}$ as a function of polymers delay line on glycerin at room temperature $\left(20^{\circ} \mathrm{C}\right)$.

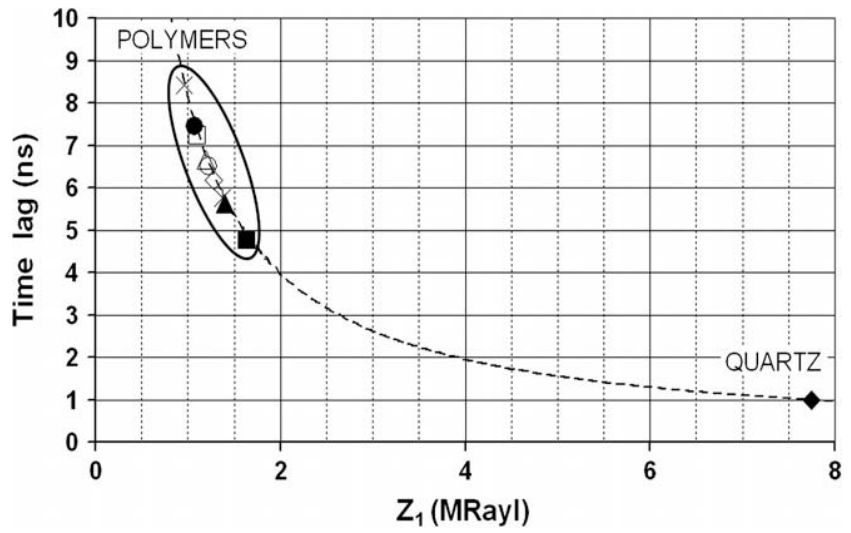

Fig. 5a. Time-lag simulation at $10 \mathrm{MHz}$ as a function of the delay line materials on glycerin at room temperature $\left(20^{\circ} \mathrm{C}\right)$.

\section{Design of the transducer}

As it is quite difficult to realise a high quality shear ultrasonic transducer, we have decided to use a commercial $10 \mathrm{MHz}$ shear transducer manufactured by Panametrics/Olympus (model V221$\mathrm{BA}$ ) and to adapt the polymer delay lines on this transducer. The main advantage is that we are sure to work with high quality shear ultrasonic waves. Indeed, one can check that for these ultrasonic transducers no longitudinal modes remain. Practically, we have

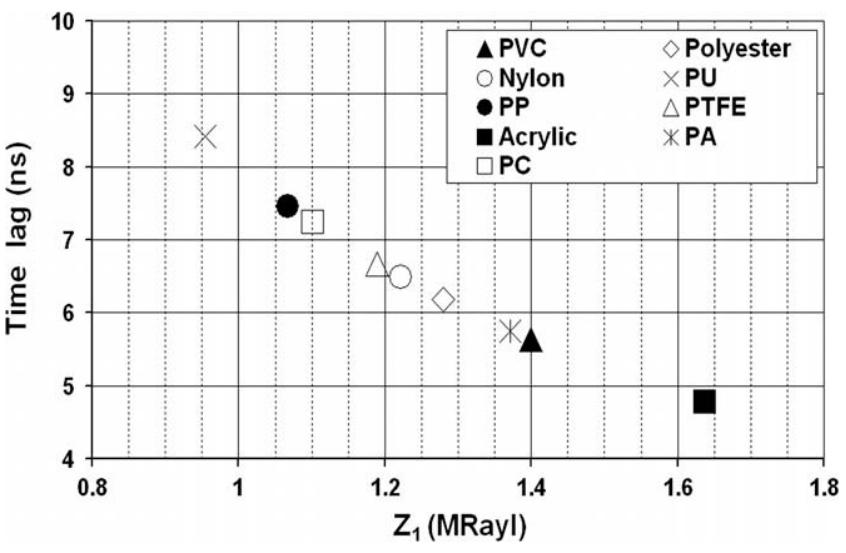

Fig. 5b. Zoom of time-lag simulation at $10 \mathrm{MHz}$ as a function of polymers delay line on glycerin at room temperature $\left(20^{\circ} \mathrm{C}\right)$.

designed plates and settled these plates on the commercial transducer. Considering the ultrasonic velocity in the plate, the thickness has been adapted for each plate in order to have a maximum of nonoverlapping echoes. For example, if we wanted to settle the acrylic plate on the commercial sensor, the thickness of the plate so is evaluated as follows: as in this study we are working with a $10 \mathrm{MHz}$ commercial ultrasonic sensor and knowing that the echoes generated in the plate have got around two periods, we have $2 T=2 / f=0.2 \mu$ s where $T$ is the period and $f$ is the frequency. So the minimal time separating two echoes has to be equal to $0.2 \mu$ s. Knowing the shear velocity (Table 1 ) and using equation (13), the appropriated thickness can be calculated. For an acrylic plate, the thickness is approximately equal to $150 \mu \mathrm{m}$. Generally, the thicknesses obtained for each polymer are around a few hundred of micrometers. As high frequency ultrasonic waves do not propagate in air, we used honey as a coupling fluid between the plate and the commercial sensor. This new system (commercial sensor + plate) has the advantage to be disposable and designed according to the working frequency. For instance, if one works with a $5 \mathrm{MHz}$ transducer the thicknesses will be multiplied by a factor 2 .

\section{Experimental setup}

The shear transducer was excited using a pulser/receiver (Panametrics Olympus $5800 \mathrm{PR}$ ) and digitized by an oscilloscope (Tektronix TDS 3032). All the measurements were made in a refrigerated incubator (BINDER KB 53) with an accuracy of $\pm 0.1^{\circ} \mathrm{C}$. The signals were recorded on a computer using a standard IEEE GPIB interface. Before each test, the free surface of the system (sensor + plate) was cleaned with ethanol. Then, it was introduced in the incubator and the reference signal (interface plate/air) was recorded. After, the material to be studied was deposited on the free surface of the system and the echoes in the delay line were again acquired. For example, we can see in the Fig. 6 the echoes recorded at the interface: "delay line/air" and "delay line/Glycerin" with a delay line made in polyurethane. As we expected, using a delay line in polymer (PU), the phase shift obtained is clearly measurable even on the first echo.

For each set of measurements, several echoes were recorded. $r_{0}$ and the phase shift $\phi$ were determined with a program elaborated under Labview $®$.

Concerning the signal processing $\left(A_{1} \ldots A_{n}\right)$ and $\left(B_{1} \ldots B_{n}\right)$ were simply evaluated using the minima and maxima on the echoes. Then, following the procedure given in [16] we plotted $\ln \left(B_{i} / A_{i}\right)$ versus $i$, for $i$ ranging from 1 to $n$. A linear adjustment of this curve directly lead to $\ln \left(r_{o}\right)$. Hence, $r_{o}$ was deduced. For the phase shift 


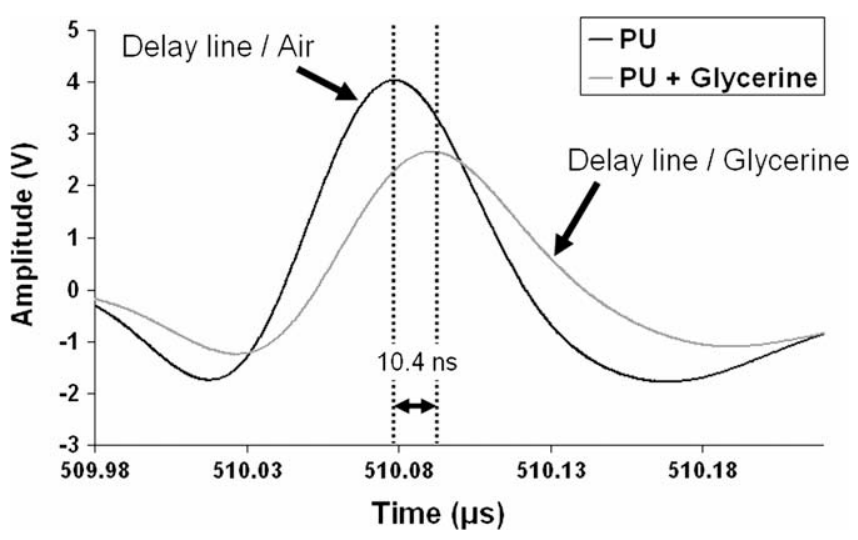

Fig. 6. Experimental echoes recorded at the interface: delay line/air and delay line/ glycerin with a delay line made in polyurethane. The measurement was realized at $10 \mathrm{MHz}$ at room temperature $\left(20^{\circ} \mathrm{C}\right)$.

evaluation, we used a cross correlation procedure [24] between the echo $i$ for the interface between delay line and air and the echo for the interface between delay line and viscoelastic material, leading to $\Delta \mathrm{t}_{i}$. Then a linear adjustment of $\Delta \mathrm{t}_{i}$ versus $i$ for $i$ ranging from 1 to $n$, lead to $\Phi$ deduction.

\section{Results}

In this experimental study, we have used the glycerin purchased from MERCK@ $\odot$. The density $(\rho)$ of the material under study provided by the manufacturer was $1260 \mathrm{~kg} \mathrm{~m}^{-3}$. For the analysis of this material, no pretreatment was necessary. One observation can be made with respect to its hygroscopic properties. The sample should be tightly closed and protected from light. Glycerin is a material known to have a Newtonian behaviour. In order to confirm this fact, we have measured the viscosity for low frequencies with a AR2000 TA Instruments Rheometer. Results are presented in Fig. 7. For each temperature ranging between 5 and $30^{\circ} \mathrm{C}$ the viscosity is not frequency dependent. This clearly demonstrates the Newtonian behaviour of this material. Thanks to a fit performed on the data (viscosity $\times$ temperature) with an Arrhenius law, we found $1.30 \pm 0.01 \mathrm{~Pa}$ s for $T=20^{\circ} \mathrm{C}$ (the error bars come from a range of repeat measurements).

The values and errors obtained for the reflection coefficient, phase shift and loss modulus as a function of the delay line material are reported in Table 2 [25]. One can notice that according to theory and to viscosity measurements, $G^{\prime \prime}$ should be equal to

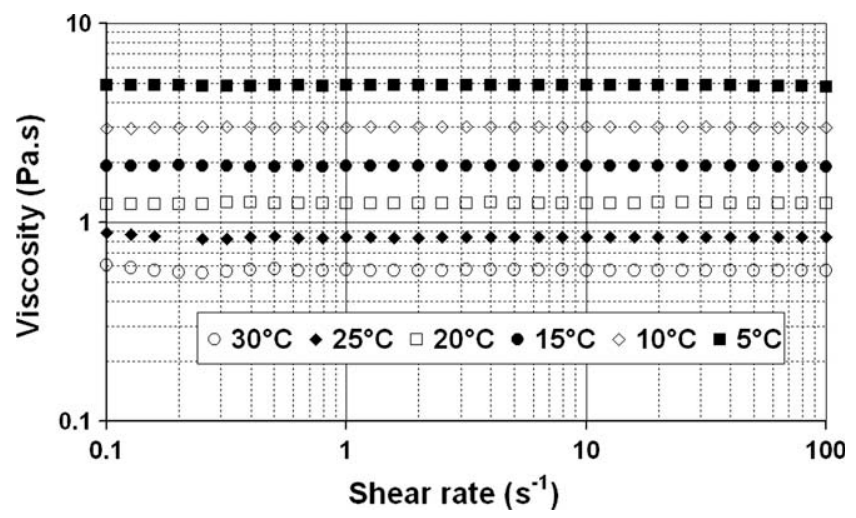

Fig. 7. Steady-state viscosity of glycerin versus shear rate for different temperatures.
Table 2

Experimental results obtained for the reflection coefficient and the time-lag at $10 \mathrm{MHz}$ as a function of the delay line material at room temperature $\left(20^{\circ} \mathrm{C}\right)$.

\begin{tabular}{lclrl}
\hline Delay line material & $Z_{1}$ (MRayl) & $\begin{array}{l}\text { Reflection } \\
\text { coefficient }\end{array}$ & \multicolumn{1}{l}{$\begin{array}{l}\text { Time-lag } \\
\text { (ns) }\end{array}$} & $\begin{array}{l}\text { G } \\
(\mathrm{MPa}) \pm 2 \%\end{array}$ \\
\hline Nylon & $1.20 \pm 0.07$ & $0.756 \pm 0.03$ & $7.50 \pm 0.14$ & 81.7 \\
Polyurethane (PU) & $0.95 \pm 0.05$ & $0.732 \pm 0.02$ & $10.40 \pm 0.20$ & 82.0 \\
Polypropylene (PP) & $1.02 \pm 0.06$ & $0.732 \pm 0.02$ & $8.80 \pm 0.17$ & 83.3 \\
Acrylic (AC) & $1.62 \pm 0.09$ & $0.792 \pm 0.03$ & $5.17 \pm 0.10$ & 82.0 \\
Polyester & $1.28 \pm 0.05$ & $0.842 \pm 0.02$ & $10.30 \pm 0.19$ & 81.5 \\
Glass & $8.36 \pm 0.04$ & $0.952 \pm 0.03$ & $0.94 \pm 0.02$ & 81.2 \\
\hline
\end{tabular}

Table 3

Comparison of real part and the modulus of the complex impedance using the experimental results obtained for the attenuation at $10 \mathrm{MHz}$ as a function of the delay line material at room temperature $\left(20^{\circ} \mathrm{C}\right)$.

\begin{tabular}{lllll}
\hline Material & $\begin{array}{l}\text { Attenuation } \\
\left(\mathrm{Np} \mathrm{m}^{-1}\right)\end{array}$ & $\begin{array}{l}\text { Real }\left[Z_{1}\right] \\
(\mathrm{MRayl})\end{array}$ & $\operatorname{Im}\left[Z_{1}\right]($ MRayl) & $\begin{array}{l}\left|Z_{1}\right| \\
(\text { MRayl })\end{array}$ \\
\hline Nylon & $353 \pm 67$ & $1.20 \pm 0.07$ & $7.06 \mathrm{E}-3 \pm 2.11 \mathrm{E}-4$ & $1.20 \pm 0.07$ \\
Polyurethane (PU) & $213 \pm 36$ & $0.95 \pm 0.05$ & $2.66 \mathrm{E}-3 \pm 1.77 \mathrm{E}-4$ & $0.95 \pm 0.05$ \\
Polypropylene (PP) & $455 \pm 86$ & $1.02 \pm 0.06$ & $8.74 \mathrm{E}-3 \pm 2.62 \mathrm{E}-4$ & $1.02 \pm 0.06$ \\
Teflon (PTFE) & $204 \pm 12$ & $1.19 \pm 0.04$ & $2.12 \mathrm{E}-3 \pm 1.02 \mathrm{E}-4$ & $1.19 \pm 0.04$ \\
Acrylic (AC) & $205 \pm 70$ & $1.62 \pm 0.09$ & $7.16 \mathrm{E}-3 \pm 2.15 \mathrm{E}-4$ & $1.62 \pm 0.09$ \\
Polyacetal (PA) & $199 \pm 51$ & $1.35 \pm 0.10$ & $4.05 \mathrm{E}-3 \pm 1.21 \mathrm{E}-4$ & $1.35 \pm 0.10$ \\
Polycarbonate (PC) & $470 \pm 52$ & $1.13 \pm 0.06$ & $7.96 \mathrm{E}-3 \pm 2.39 \mathrm{E}-4$ & $1.13 \pm 0.06$ \\
Polyester & $409 \pm 61$ & $1.28 \pm 0.05$ & $9.68 \mathrm{E}-3 \pm 2.90 \mathrm{E}-4$ & $1.28 \pm 0.05$ \\
\hline
\end{tabular}

81.7 MPa because $G^{\prime \prime}=\omega \eta$. The results given in Table 2 are in good agreement with this value.

Remark 1: one can notice that the material with best sensitivity (PU) does not seem to give the best result compared to glass. However experiments with glass are more difficult because one has to use many echoes (over 10) which have smaller and smaller amplitudes during propagation.

Remark 2: here results are presented at $20^{\circ} \mathrm{C}$. But other measurements at various temperatures gave the same agreement between rheological measurements and ultrasonic approach. For instance, for $T=10^{\circ} \mathrm{C}$ the viscosity measured with the rheometer is $3 \pm 0.01 \mathrm{~Pa}$. With ultrasound we found $185 \pm 4 \mathrm{MPa}$ for $f=10 \mathrm{MHz}$ leading to viscosity of $2.94 \pm 0.06$ according to $G^{\prime \prime}=\omega \eta$.

As predicted by theory (Figs. 2 and 3 ), the best sensitivity is given for the polymer delay line with the lowest acoustic impedance, in our case, the delay line in polyurethane. We can observe that, if we use a delay line in polyurethane instead of quartz, the sensitivity of the system can be multiplied by 10 .

In this study, we considered that the materials used as delay line have a low attenuation and therefore this attenuation could be neglected. However, polymers are known as ultrasonic absorbent materials. In order to verify the error associated with the calculation of $G^{\prime \prime}$, we will now use the theory taking into account the complex impedance. In the next section, this point will be discussed.

\section{Discussion}

To take into account the complex impedance theory and calculate the parameters $b$ and $c$, we need to know the attenuation of each plate used as a delay line. To determine the attenuation in these materials, we have made various plates of each material in different thicknesses. Thanks to the measurement of the amplitude of a transmitted signal, the attenuation was easily deduced. The values obtained for the attenuation and the comparison between the real and the modulus of the complex impedance are reported in Table 3. 
As we can see, the imaginary part of complex impedance can be neglected when compared to the real part. In the case of viscoelastic materials, these low attenuations may seem surprising but at room temperature we are investigating a zone after the glass transition. Hence, $G^{\prime} \gg G^{\prime \prime}$ and viscous behaviour is largely inferior to the elastic one. Consequently, the attenuation in the delay line is small. So, considering a real acoustical impedance for the delay lines in polymers was acceptable in the context of this work.

\section{Conclusions}

Using simple plates in polymers settled on a commercial shear ultrasonic sensor we have demonstrated that the sensitivity of our piezo rheometer could be multiplied by a factor 10 . Hence, with a very robust and simple system we obtain a sensitivity at least equal to more complex system using oblique incidence. The validation on glycerin also shows that the accuracy of the system is very good.

For the future, it will be advantageous to build commercial sensors by depositing thin polymer films onto the transducer element. This will allow a perfect coupling and consequently, the generation of a maximum number of echoes. An application of such sensors on classical rheometers could be interesting in order to obtain in the same measurement low and high frequency behaviour of viscoelastic products.

\section{References}

[1] J.P. Ferry, Viscoelastic Properties of Polymers, Wiley, New York, 1980.

[2] W.P. Mason, Physical Acoustics, vol. 1, Academic Press, New York, 1964 (Part A).

[3] A.W. Chow, J.L. Bellin, Simultaneous acoustic wave propagation and dynamic mechanical analysis of curing of thermoset resins, Polymer Engineering and Science 32 (3) (1992) 182-190.

[4] R.J. Freemantle, R.E. Challis, Combined compression and shear wave ultrasonic measurements on curing adhesives, Measurement Science and Technology 9 (1998) 1291-1302.

[5] M. Frigone, A. Maffezzoli, D. Acierno, V.A.M. Luprano, G. Montagna, Nondestructive and in-situ monitoring of mechanical property buildup in epoxy adhesives for civil applications by propagation of ultrasonic waves, Polymer Engineering and Science 40 (3) (2000) 656-664.

[6] I. Alig, M. Fedtke, K.-G. Häusler, W. Tänzer, S. Wartewig, Modified epoxies as studied by ultrasonic methods, Progress in Colloid and Polymer Science 78 (1988) 54-58.
[7] I. Alig, S. Tadjbakhsch, A. Zosel, Comparison of ultrasonic shear wave and dynamic-mechanical measurements in acrylic-type copolymers, Journal of Polymer Science. Part B: Polymer Physics 36 (1998) 1703-1711.

[8] I. Alig, D. Lellinger, Ultrasonic methods for characterizing polymeric material, Chemical Innovation 30 (2) (2000) 12-18.

[9] I. Alig, D. Lellinger, J. Sulimma, S. Tadjbakhsch, Ultrasonic shear wave reflection method for measurements of the viscoelastic properties of polymer films, Review of Scientific Instruments 68 (3) (1997) 1536-1542.

[10] Z. Sun, T. Voigt, S.P. Shah, Rheometric and ultrasonic investigations of viscoelastic properties of fresh Portland cements pastes, Cement and Concrete Research 36 (2006) 278-287.

[11] H.T. O'Neil, Reflection and refraction of plane shear waves in viscoelastic media, Physical Review 75 (6) (1949) 928-935.

[12] W.P. Mason, W.O. Baber, H.J. McSkimin, J.H. Heiss, Measurement of shear elasticity and viscosity of liquids at ultrasonic frequencies, Physical Review 75 (6) (1949) 936-946.

[13] A. Yoneda, M. Ichihara, Shear viscoelasticity of ultrasonic couplers by broadband reflectivity measurements, Journal of Applied Physics 97 (2005) 054901.

[14] P.Y. Longin, C. Verdier, M. Piau, Dynamic shear rheology of high molecular weight polydimethylsiloxanes: comparison of rheometry and ultrasound, Journal of Non-Newtonian Fluid Mechanics 76 (1998) 213-232.

[15] R.E. Challis, M.E. Unwin, D.L. Chadwick, R.J. Freemantle, I.K. Partridge, D.J. Dare, P.I. Karkanas, Following network formation in an epoxy/amine system by ultrasound, dielectric and nuclear magnetic resonance measurements: a comparative study, Journal of Applied Polymer Science 88 (2003) 1665-1675.

[16] J. Gasparoux, D. Laux, J.Y. Ferrandis, J. Attal, P. Tordjeman, Large frequency bands viscoelastic properties of honey, Journal of Non-Newtonian Fluid Mechanics 153 (1) (2007) 46-52.

[17] S. Goto, H. Nagazono, H. Kato, The flow behavior of fiber suspensions in Newtonian fluids and polymer solutions. I. Mechanical properties, Rheologica Acta 25 (1986) 119-129.

[18] Ir.R. Rutgers, Relative viscosity of suspensions of rigid spheres in Newtonian liquids, Rheologica Acta 2 (3) (1962) 202-210.

[19] H. Miura, T. Takahashi, Y. Kawase, Effect of pseudoplastic behaviour of liquid in co-current three-phase fluidized beds on bed expansion, Chemical Engineering Science 56 (2001) 6047-6053.

[20] K. Schröter, E. Donth, Viscosity and shear response at the dynamic glass transition of glycerol, The Journal of Chemical Physics 113 (20) (2000) 9101.

[21] Jong-Wooka Ha, Seung-Man Yang, Deformation and breakup of Newtonian and non-Newtonian conducting drops in an electric field, Journal of Fluid Mechanics 405 (2000) 131-156.

[22] C.R. Wildemuth, M.C. Williams, Viscosity of suspensions modeled with a shear-dependent maximum packing fraction, Rheologica Acta 23 (6) (1984) 627-635.

[23] D. Lootens, Céments et suspension concentrées modèles, écoulement, encombrement et floculation, PhD thesis, 2004.

[24] D.R. Hull, H.E. Kautz, A. Vary, Measurements of ultrasonic velocity using phase-slope and cross-correlation methods, Material Evaluation 43 (1985) $1455-1460$.

[25] R.E. Challis et al., Errors and uncertainties in the pulse-echo reflectometry method for measuring acoustic impedance, Measurement Science and Technology 9 (1998) 692-700. 